\title{
Abrupt turn-on and hysteresis in a VCSEL with frequency-selective optical feedback
}

\author{
Alexander Naumenko, Natalia Loiko \\ Institute of Physics, Academy of Sciences of Belarus, \\ Scaryna Prospekt 70, 220072 Minsk, Belarus
}

\author{
Markus Sondermann, Karl Jentsch, Thorsten Ackemann \\ Department of Physics, University of Strathclyde, \\ 107 Rottenrow, Glasgow G4 ONG, Scotland, UK; formerly at: Institute for \\ Applied Physics, University of Münster
}

\begin{abstract}
The emission characteristics of a vertical-cavity surface-emitting laser (VCSEL) operated in a single-transverse mode and coupled to an external cavity with a diffraction grating as a frequency-selective element are analyzed experimentally, numerically and analytically. The experiments yield a rather abrupt turn-on of the VCSEL to a high-amplitude emission state and hysteresis phenomena. The experimental results are explained by numerical simulations and analytical calculations demonstrating the possibility of bistability between lasing and non-lasing states close to threshold. Hence, the scheme might be useful in all-optical photonic switching applications. A detailed bifurcation analysis near threshold is given by superimposing the numerical results with analytical steady-state curves. The mode selection and switching behavior obtained in the simulations can be interpreted from the point of view of the preference of states with the minimal total losses.
\end{abstract}

Key words: VCSEL, filtered feedback, external cavity, polarization, switching PACS: 42.55.Px, 42.60.Mi, 42.65.Pc, 42.65.Sf

Email addresses: n.loiko@dragon.bas-net. by (Natalia Loiko), thorsten.ackemann@strath.ac.uk (Thorsten Ackemann). 


\section{Introduction}

This paper is devoted to investigations of the influence of external filtered optical feedback on the operation of a vertical-cavity surface-emitting laser (VCSEL). Frequency-selective optical feedback was suggested to stabilize singlelongitudinal mode operation of edge-emitting laser diodes. Several filtering schemes were realized on basis of a narrow-band resonator [1], a grating [2], a fiber Bragg grating reflector [3] or by locking the frequency of the diode to transition lines of a medium placed within the external cavity [4]. Along with a stable single-mode lasing characteristics, more complex phenomena as multistability, hopping between internal modes and dynamical behaviors including the phenomenon of low-frequency fluctuations were found (see for example [5-12]).

In contrast to conventional edge-emitting semiconductor lasers, VCSELs operate intrinsically in a single longitudinal mode. However, their cylindrical geometry results in a rather weak intrinsic polarization selection. As a result, the phenomenon of polarization switching is often observed when the pump current is varied [13-16]. One of the techniques to control the polarization of VCSELs is the use of external anisotropic optical feedback [17-21]. Frequency selective optical feedback with an appropriate bandwidth could also influence the polarization selection due to the frequency splitting between the two sets of the external cavity modes with orthogonal polarization. In addition, a diffraction grating provides polarization selectivity, since the diffraction efficiency of a grating depends on the angle between the grooves and the electrical field vector of the incident field. Combination of frequency and polarization selection properties in one device can open new opportunities for control of VCSEL operation. This provides one main motivation for our experimental and theoretical investigations of a VCSEL coupled to an external cavity containing a diffraction grating as a frequency selective element. The anisotropy property of a grating have been investigated recently in a monolithically integrated configuration [22]. The main difference of a laser scheme with diffraction grating inserted in a delayed external cavity is that the last provides a competition between external cavity modes that enriches the laser behaviors and introduces new switching phenomena.

A second motivation for our studies comes from the fact that frequencyselective feedback is known to favor the possibility of bi- or multi-stability between different emission states (see, e.g., $[6,8,9,11,12]$ for edge-emitting lasers). In particular, we will consider a new type of bistability between lasing and non-lasing states and the resulting abrupt turn-on of the laser. These phenomena appear due to the interplay of the frequency selectivity of the grating, phase-amplitude coupling and thermal shifts of the emission frequency. This bistability provides potential for all-optical switching in photonic applications. 


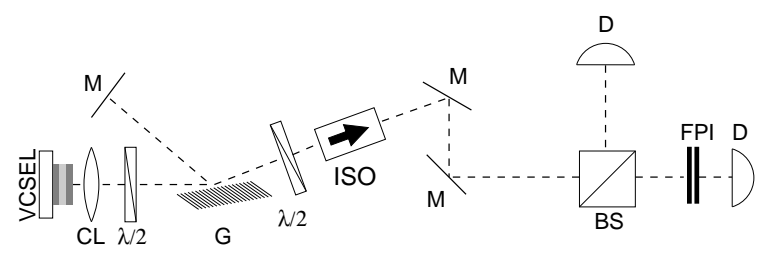

Fig. 1. Experimental setup: CL collimating lens, $\lambda / 2$ half-wave retardation plate, G grating, M HR-mirror, ISO optical isolator, BS beam splitter, D low-bandwidth detector, FPI scanning Fabry-Perot interferometer.

In the case of broad-area devices, bistability could be favorably explored to excite spatial localized emission states (cavity solitons) by an external focused control beam (cf. to [29] for preliminary results). Cavity solitons are considered to be attractive as "bits" for future all-optically and potentially massively parallel information processing schemes [23]. It is worth noting that a similar setup was used in [24] for the transverse-mode control in a VCSEL.

\section{Experiments}

The experimental setup is shown in Fig. 1. The VCSELs investigated are gainguided devices with an aperture diameter of $15 \mu \mathrm{m}$ from EMCORE Corp. (emission wavelength in the $850 \mathrm{~nm}$ region) operating in the fundamental transverse mode close to threshold (up to approx. $20 \%$ above threshold). The light is collected by an aspheric, anti-reflection coated lens. The external cavity is set up in Littman configuration, i.e., a holografic reflection grating (2000 lines $/ \mathrm{mm}$, Au coated) is hit at grazing incidence and the first diffraction order is retro-reflected by a plane highly-reflecting mirror. The bandwidth of the bandpass is estimated to be about 20-30 GHz. The zeroth order is coupled out and used for detection. The length of the external cavity is about $21 \mathrm{~cm}$.

Since in general the principal axes of the polarization ellipse of the VCSEL are not parallel, respectively orthogonal, to the grating grooves, a half-wave plate is inserted in the external cavity, which is oriented such that the principal axes of the light field match the principal axes of the grating. Due to the fact that the diffraction efficiency of the grating is highly anisotropic (if the electrical field vector is orthogonal to the grooves, the efficiency is more than five times higher than if it is parallel), also a polarization selectivity is obtained in the feedback. The highest achievable threshold reduction is about $10 \%$ obtained by adjusting the tilt of the external mirror and the position of the collimation lens.

The laser is isolated from the detection path by an optical isolator with an isolation of more than $60 \mathrm{~dB}$. A low-bandwidth detector is used to monitor the time-averaged, polarization resolved light-current (LI-) characteristics. The 


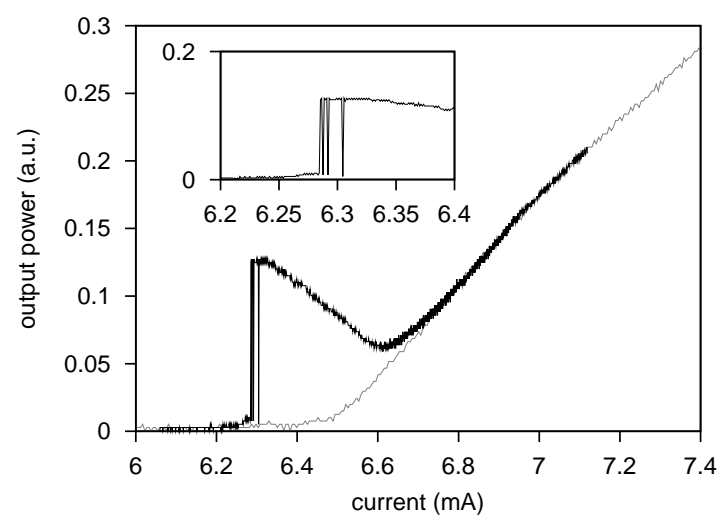

Fig. 2. Output power for increasing injection current. The anisotropies of the external cavity are optimized for feedback favoring the polarization component already dominant in the free-running laser. The grey line denotes the free running laser, the black one the laser with feedback. The inset shows an enlargement of the region around threshold. The detuning is optimized to achieve a large response in the vicinity of the threshold.

polarization resolved optical spectrum is measured with a scanning FabryPerot interferometer with a finesse of 200 and a free spectral range of $46 \mathrm{GHz}$.

The free-running laser has a threshold of about $6.45 \mathrm{~mA}$. The LI-curve is approximately linear and the turn-on behavior is continuous (see grey line in Fig. 2). This is a usual behavior of a laser, which shows a supercritical bifurcation from the non-lasing to the lasing state. One of the linear polarization components is strongly dominating the emission, which is the mode with higher frequency in the device considered here.

In a first experiment, the axis of the retardation plate is adjusted such that the polarization already dominant in the free-running laser benefits more strongly from the feedback. The 'grating frequency' (i.e., the frequency with the highest feedback rate due to the combined action of the angular dispersion of the grating and the adjustment of the feedback mirror) is tuned close to the operating frequency of the free-running laser at threshold by adjusting the horizontal tilt controls of the feedback mirror. If all other parameters are optimized for good feedback efficiency (i.e., the vertical tilt control of the external mirror and the position of the collimation lens), a very abrupt switch-on of the emission power of the laser is found (see black line in Fig. 2) after a first, very small pedestal, if the injection current is increased. After the switch-on, there is a small current range (see inset of Fig. 2) in which the laser switches - apparently erratically - down to very low power again and recovers very fast to the high power level. Both features observed - the abrupt switch-on to a finite power-level and the existence of power drops with the maximum modulation depth - hint to the existence of bistability between a lasing state of finite amplitude and the non-lasing state. 


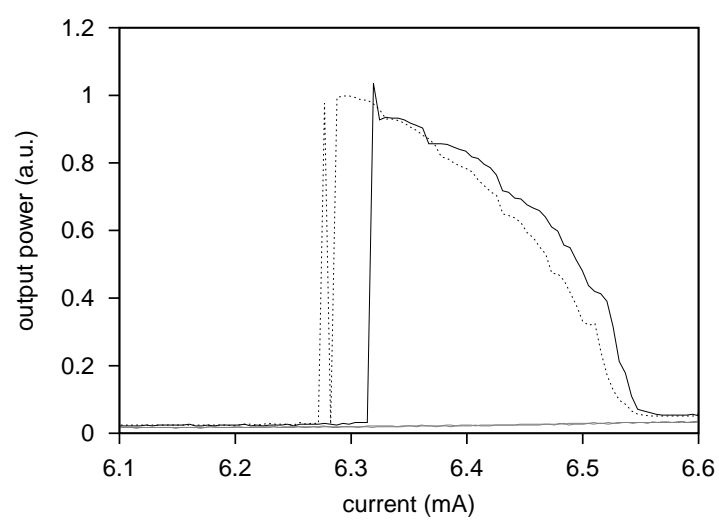

Fig. 3. Output power in dependence on injection current. The anisotropies of the external cavity are optimized for feedback favoring the polarization component disfavored in the free-running laser. The grey line denotes the free running laser, the black solid line the laser with feedback for increasing current, the black dotted line the laser with feedback for decreasing current. The detuning is optimized to achieve a large response in the vicinity of the threshold.

If the current is increased further, the output power decreases until a local minimum is reached at about $6.6 \mathrm{~mA}$. Afterwards the output power increases monotonically with current again and approaches asymptotically the value of the free-running laser. This indicates that the feedback strength decreases and actually vanishes for currents larger than about $7 \mathrm{~mA}$. This behavior will be explained in the following sections by the fact that the frequency of the free-running laser shifts due to ohmic heating, if the current is increased (with a rate of about $-50 \mathrm{GHz} / \mathrm{mA}$ ) and hence the laser frequency is shifted in resonance for low currents (at $6.3 \mathrm{~mA}$, causing bistability) and leaves the resonance again for about 6.7-6.8 $\mathrm{mA}$.

The grey line in the very bottom of Fig. 3 shows the experimental LI-characteristics of the polarization mode disfavored in the free-running laser without feedback. If the retardation plate is adjusted such that this mode is favored by the feedback, a rather large signal with an abrupt switch-on can be obtained also for this mode (solid black line in Fig. 3). As discussed for the orthogonal mode (see above), the output decreases, if the current is increased. At about $6.5 \mathrm{~mA}$ it stabilizes at a level, which is slightly larger than the one of the free-running laser. It remains on that level within the regime of fundamental-mode operation.

Fig. 3 contains also the LI-characteristics for decreasing current. For values above $6.32 \mathrm{~mA}$, it follows quite closely the curve of the up-scan, but the laser stays on beyond the switch-on point, before it switches off. The hysteresis loop has a width of about $0.04 \mathrm{~mA}$. This and the intermediate drop-out at $6.28 \mathrm{~mA}$ confirm again the subcritical character of the bifurcation and the existence of bistability. 


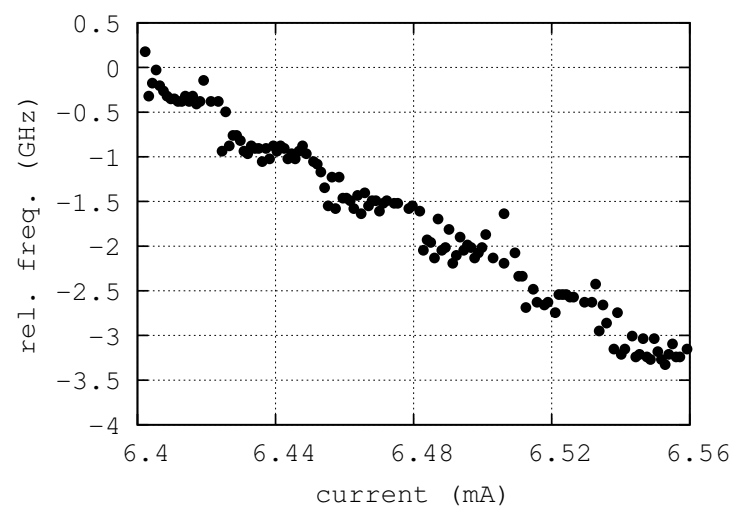

Fig. 4. Emission frequency versus current in the grating controlled regime of Fig. 3.

Above threshold, the LI-characteristic has some small amplitude 'wiggles', which are more pronounced in the down-scan than in the up-scan. Fig. 4 shows the emission frequency as measured in the optical spectrum in dependence on current in the grating controlled regime. Especially for lower currents, a tendency for a stair-case like 'grouping' of frequencies is apparent. We interpret this as the locking at external cavity modes followed by switching events between these modes causing also the ripples in the LI-curve. The slope of the curve is $20 \mathrm{GHz} / \mathrm{mA}$, i.e., considerably smaller than the current-induced frequency shift of the free-running laser.

The above results motivate corresponding theoretical investigations in order to elucidate the reasons for the observed phenomena, especially the existence of an apparently subcritical turn-on behavior.

\section{Theoretical model}

The so called spin-flip-model (SFM) of San Miguel and coworkers was chosen to simulate the polarization and spin dynamics of a VCSEL based on a quantum-well active medium [25]. The modified rate equations describing the polarization dynamics in a VCSEL with optical feedback from an external reflector have a form similar to the one formulated in [19]

$$
\begin{aligned}
& \frac{d}{d t} \bar{E}=\hat{G} \cdot \bar{E}+\bar{F}+\bar{W}_{E}, \\
& \frac{d}{d t} \bar{N}=\bar{\mu}+\hat{S} \cdot|\bar{E}|^{2}-\bar{W}_{N}
\end{aligned}
$$

where $N_{ \pm}=N \pm n, \gamma=\gamma_{a}+i \gamma_{p}$, column vectors are denoted ' ${ }^{-}$': $\bar{E} \equiv$ $\left(E_{+}, E_{-}\right),|\bar{E}|^{2} \equiv\left(\left|E_{+}\right|^{2},\left|E_{-}\right|^{2}\right), \bar{N} \equiv(N, n), \bar{\mu}=\left(\Gamma(\mu-N),-\gamma_{s} n\right)$; while 
matrices are denoted '^':

$$
\begin{aligned}
& \hat{G}=\left(\begin{array}{cc}
\kappa(1+i \alpha)\left(N_{+}-1\right)+i \Delta \omega_{0} & -\gamma \\
-\gamma & \kappa(1+i \alpha)\left(N_{-}-1\right)+i \Delta \omega_{0}
\end{array}\right), \\
& \hat{S}=-\Gamma\left(\begin{array}{c}
N_{+} N_{-} \\
N_{+}-N_{-}
\end{array}\right) .
\end{aligned}
$$

$E_{ \pm}=\frac{1}{\sqrt{2}}\left(E_{x} \pm i E_{y}\right)$, where $E_{x}$ and $E_{y}$ are $\hat{x}$ - and $\hat{y}$ - polarized components of the slowly varying amplitude of the electromagnetic field; $N$ is the total population difference between the conduction and valence bands; $n$ is the difference of the population differences for the two allowed transitions between magnetic sublevels; $\kappa$ is the mean of the decay rates of the two linearly polarized components of the field; $\alpha$ is the linewidth enhancement factor; $\gamma_{a}$ is the anisotropic field loss rate (positive $\gamma_{a}$ gives the $\hat{y}$ - polarized component a lower threshold); $\gamma_{p}$ represents the linear birefringence of the medium (which gives opposite frequency shifts for the different linearly polarized fields of the solitary laser); $\mu$ is the normalized injection current, which takes the value 1 at the solitary laser threshold; $\gamma_{s}$ is the decay rate for the difference in the populations of the different magnetic sublevels which is reduced by both spontaneous emission and spin-flip relaxation processes; $\Gamma$ is the decay rate of the total carrier population (we use a typical value of $1 / \Gamma \equiv \tau_{e}=1 \mathrm{~ns}$ ). We introduce here the detuning $\Delta \omega_{0}$ of the solitary laser frequency from its reference value $\omega_{0}$ due to the thermal effects, and the following phenomenological dependence of this detunig on the injection current is used (see, e.g., $[11,12]$ ):

$$
\Delta \omega_{0}=\Delta \omega_{0}^{t h}-2 \pi k_{\mu}(\mu-1),
$$

where $\Delta \omega_{0}^{t h}$ is the value of the detuning at the onset of lasing and the coefficient $k_{\mu}$ can be defined from experiments. The effect of filtered feedback from a diffraction grating is calculated in Fourier space:

$$
\bar{F}(t)=\frac{1}{2 \pi} \int_{-\infty}^{+\infty} d \omega \hat{K}(\omega) \int_{-\infty}^{+\infty} d t^{\prime} \bar{E}\left(t^{\prime}\right) \exp \left(i \omega\left(t-t^{\prime}\right)\right)
$$

where $\hat{K}(\omega)$ is a full matrix transfer function of the external cavity. Here, for simplicity, we take into account only one field round-trip in the external cavity. As discussed in the experimental section, the polarization principal axis of the grating are aligned with the ones of the VCSEL, but the diffraction efficiency is anisotropic. Hence, the transfer function can be represented as:

$$
\hat{K}(\Omega)=\hat{\sigma} h(\Omega)^{2} \exp \left(-i \tau\left(\Omega+\omega_{0}\right)\right),
$$


where the square is due to the double reflection of the light from the grating in the considered experimental configuration, $\tau$ is the external cavity round-trip time, and

$$
\hat{\sigma}=\frac{1}{2}\left(\begin{array}{c}
\sigma_{x}+\sigma_{y} \sigma_{x}-\sigma_{y} \\
\sigma_{x}-\sigma_{y} \sigma_{x}+\sigma_{y}
\end{array}\right) .
$$

Here, $\sigma_{x, y}$ represent the feedback strength for the x- and y- polarized components, where $\sigma_{x, y}=\left(1-r_{2}^{2}\right) r_{x, y}^{2} /\left(r_{2} \tau_{i n}\right) \cdot r_{2}$ is the amplitude reflectivity of the outcoupling mirror of the VCSEL and $r_{x, y}$ are the corresponding values for the grating (on peak) for the two orthogonal polarization components.

$h(\Omega)$ is a normalized frequency dependent part of the transfer function (or a normalized reflection coefficient) of the grating, which is given by (see appen$\operatorname{dix}$ A.1)

$$
h(\Omega)=\exp \left(-i T\left(\Omega-\omega_{m}\right)\right) \frac{\sin T\left(\Omega-\omega_{m}\right)}{T\left(\Omega-\omega_{m}\right)} .
$$

Here, $\omega_{m}$ is the frequency of the main grating maximum (more exactly, it is the detuning from the frequency reference $\left.\omega_{0}\right), 1 / T$ is the grating bandwidth. The transfer function (8) corresponds to a Green function (or an impulse response function) with a rectangular shape in the time domain $\left(h(\Omega)^{2}\right.$ with a triangular shape) [12]. For the sake of convenience of numerical simulations, we reformulate the above integral equation for the feedback term $\bar{F}(t)$ as differential equations with delay terms (in a symmetrical form):

$$
\begin{aligned}
& \frac{d}{d t} \bar{F}_{1}(t)=\frac{1}{2 T}\left[\bar{E}(t-\tau / 2)-\bar{E}(t-\tau / 2-2 T) \exp \left(i \omega_{m} T\right)\right]+i \omega_{m} \bar{F}_{1}(t), \\
& \frac{d}{d t} \bar{F}_{2}(t)=\frac{1}{2 T}\left[\bar{F}_{1}(t-\tau / 2)-\bar{F}_{1}(t-\tau / 2-2 T) \exp \left(i \omega_{m} T\right)\right]+i \omega_{m} \bar{F}_{2}(t) .
\end{aligned}
$$

Then, $\bar{F}(t)=\hat{\sigma} \exp \left(-i \omega_{0} \tau\right) \bar{F}_{2}(t)$.

Langevin noise sources $\bar{W}_{E} \equiv\left(W_{+}, W_{-}\right)$and $\bar{W}_{N} \equiv\left(W_{N}, W_{n}\right)$ that arise from spontaneous emission processes have the following form: $W_{ \pm}=\sqrt{\beta_{n} \Gamma N_{ \pm}} \xi_{ \pm}(t)$ and $W_{(N, n)}=\frac{\Gamma}{2 \kappa}\left(W_{+}^{*} E_{+} \pm W_{-}^{*} E_{-}+\right.$c.c. $)$, where $\beta_{n}$ is the spontaneous emission factor (the fraction of the spontaneously emitted photons that goes into the lasing modes); $\xi_{ \pm}$are two independent complex noise sources with zero mean and the correlation $\left\langle\xi_{ \pm}(t) \xi_{ \pm}^{*}\left(t^{\prime}\right)\right\rangle=2 \delta\left(t-t^{\prime}\right)$. In numerical simulations they are represented as $\xi_{ \pm}(t)=\chi_{ \pm} / \sqrt{\Delta t}$, where $\chi_{ \pm}$are complex Gaussian random variables (with zero mean and standard deviation $\left\langle\chi_{i} \chi_{i}^{*}\right\rangle=2$ ), and $\Delta t$ is the time interval over which the noise is held constant. 
The values of the parameters used in the numerical simulations are: $\kappa=$ $300 n s^{-1}, r_{2}=0.9975$ and $\tau_{i n}=28 \mathrm{fs}, \alpha=5, \gamma_{a}=0.5 \pi n s^{-1}, \gamma_{p}=25 \pi n s^{-1}$, $\gamma_{s}=40 n s^{-1}, \sigma_{x}=60 n s^{-1}, \sigma_{y}=10 n s^{-1}, \tau=1.4 n s, \beta_{n}=10^{-5}, \Delta t=10 p s$, $k_{\mu}=600 \mathrm{GHz}$ (corresponding to a frequency drift of $100 \mathrm{GHz} / \mathrm{mA}$ for a threshold current of $J_{t h}=6 \mathrm{~mA}$ ), HWHM $=24 \pi \mathrm{GHz}$, though different values for the parameters have been also taken for calculations. A good qualitative agreement with experiments is observed when the ratio between the bandwidth of the filter and the rate of the frequency drift is close to the experimental one.

The stationary states of the model equations and their stability analysis are given in appendixes A.2 and A.3. For a numerical integration of the time evolution of the model equations, a fourth-order variable-step method was used. Intensive investigations of the hysteresis phenomena in the polarization resolved LI-curves and of polarization dynamics have been performed. Moreover, transfer functions corresponding to a single reflection from the grating, to a Lorentzian filter and to frequency independent feedback have been considered for comparison. Differences in the filtering schemes influence primarily only the quantitative aspects but not the qualitative features of the phenomena described below.

\section{Numerical results and comparison with experiments}

\subsection{Feedback conditions favoring the weaker polarization mode}

First, we are going to consider a situation, where the anisotropy of the feedback conditions is adjusted in such a way that the polarization component, which was disfavored in the solitary laser, profits more from the feedback. Concretely, the feedback favors the lower frequency (x-polarized) component having higher losses in the solitary laser, i.e., the sign of the birefringence and of the dichroism is positive. The detuning between the laser frequency at the threshold of the solitary laser and the grating frequency is taken to be zero. For this situation, Fig. 5b shows the steady state mode structure of the VCSEL with filtered feedback in the domain frequency-intensity. For comparison, Fig. 5a shows the case of feedback without filtering in frequency space, but including the polarization anisotropy.

The steady-states are linearly polarized. The set of steady-states belonging to each of the two polarization components has the same properties as the scalar steady-states of an edge-emitting laser with filtered feedback (see, e.g., [10,12] and Refs. therein), i.e., in the $|E|-\Omega$-plane it forms a curve - sometimes referred to as the fixed-point ellipse - comprised by external cavity modes and 
(a)
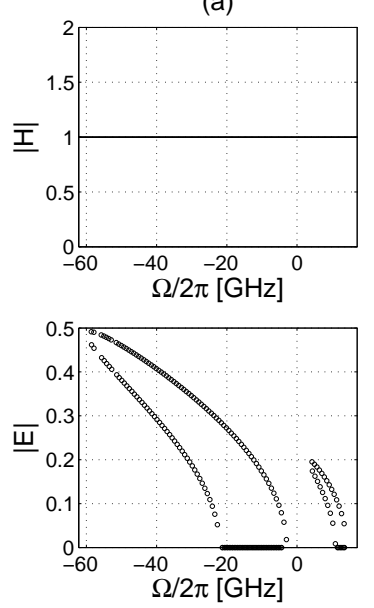

(b)
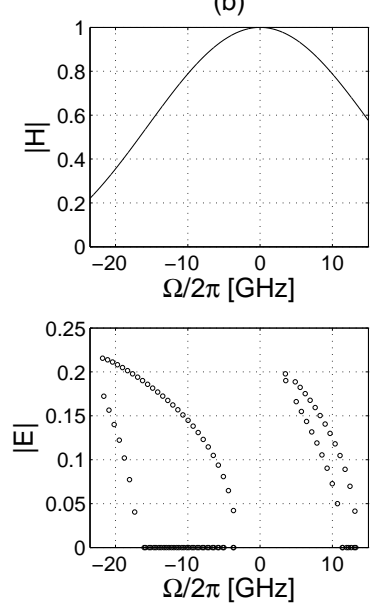

Fig. 5. Steady states (external cavity modes) in the domain frequency-amplitude (lower panels) without frequency-filtering effects (a) and with frequency-filtering from the external diffraction grating (b) and corresponding transfer functions (upper panels). Parameters: $\kappa=300 n s^{-1}, \alpha=5, \gamma_{a}=0.5 \pi n s^{-1}, \gamma_{p}=25 \pi n s^{-1}$, $\gamma_{s}=40 n s^{-1}, \mu=1, \tau=1.4 n s, \sigma_{x}=60 n s^{-1}, \sigma_{y}=10 n s^{-1}, H W H M=24 \pi n s^{-1}$, $\Delta \omega_{0}=0, \omega_{m}=0$.

anti-modes. Since the threshold condition at low injection currents is satisfied only for the part of the external cavity modes having the higher gain, the ellipses are not closed but cut off at zero intensity.

The (half-) ellipses for the $x$ - and $y$-components are shifted with respect to each other due to the birefringence. Without filtering, the resulting half-ellipses have different size for the two polarization components determined by the intrinsic and external polarization anisotropies. Here, the net effect favors the $x$-polarized mode. With filtering, also the difference in detuning to the grating frequency for the two polarization components plays a strong role, which is due to the birefringence. Hence, in the presence of frequency-selective feedback one of the two polarization modes can be strongly favored due to the effect of birefringence, even if it is disfavored by dichroism. Here, the y-polarized EC-modes with maximal gain are in the vicinity of the grating frequency. Hence, their amplitude does not change very much in comparison to the case without filtering. In contrast, the $x$-polarized EC-modes with the highest gain are strongly affected and have a considerably lower amplitude because they are red-shifted. For the same reason, also the number of $x$-polarized external cavity modes is strongly decreased. Hence, favorable amplitude anisotropies can be compensated by unfavorable phase anisotropies (birefringence).

We will study the interplay of the finite bandwidth of the grating, the thermal frequency shift and the birefringent splitting between the two polarization components in more detail below. Similar as in a 'scalar laser' [10,11], an increase of the current does not necessarily lead to an increase of output 

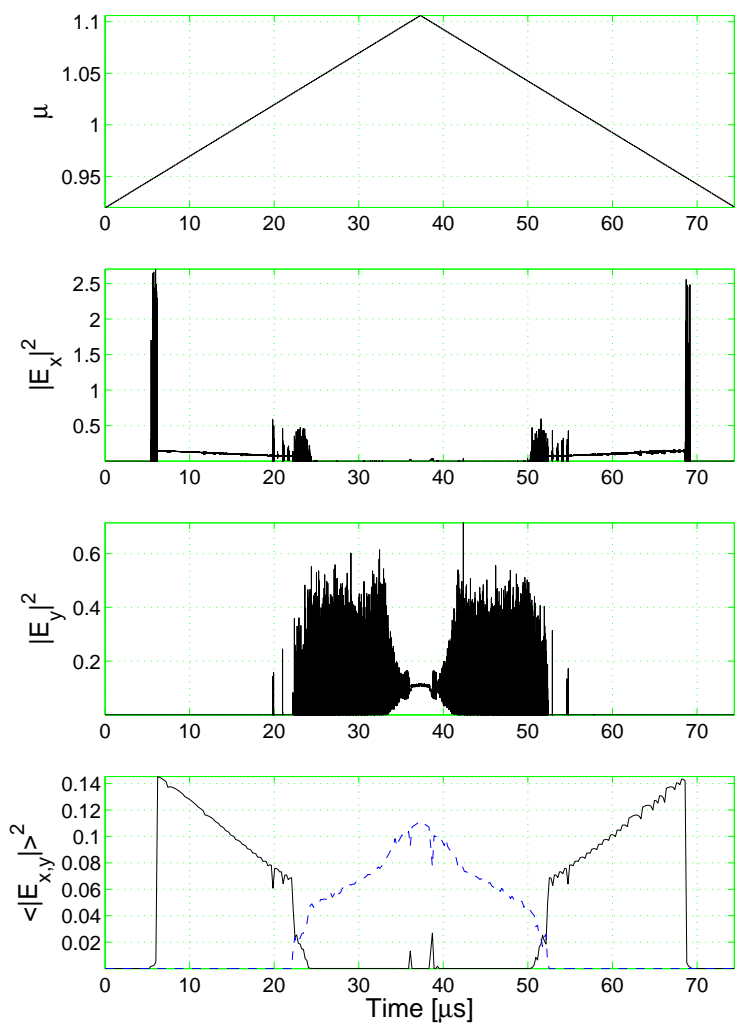

Fig. 6. Time evolution of instantaneous intensities of $E_{x^{-}}$and $E_{y^{-}}$polarized components $\left(\left|E_{x}\right|^{2}\right.$ and $\left|E_{y}\right|^{2}$, central panels) and of averaged intensities $\left(<\left|E_{x}\right|>^{2}\right.$ (solid curve) and $\left\langle\left|E_{y}\right|\right\rangle^{2}$ (dashed curve), lowermost panel), when the injection current $\mu$ is scanned (uppermost panel). Parameters: $\beta=10^{-5}, \Delta \omega_{0}^{\text {th }}=30 \pi G H z$, $k_{\mu}=600 \mathrm{GHz}$. Other parameters are the same as in Figure 5.

amplitude since the laser frequency might leave the effective bandwidth of the grating. This effect is obvious in the experimental measurements (Figs. 2, 3) for both polarized components.

First, we assume a positive detuning between the solitary laser threshold frequency and the grating frequency $\left(\Delta \omega_{0}^{t h}-\omega_{m}=30 \pi \mathrm{GHz}\right)$. Starting from a value of the injection current below threshold, the current is increased step by step up to $11 \%$ above the threshold of the solitary laser (taking into account the thermal frequency-shift, $k_{\mu}=600 \mathrm{GHz}$ ) and then decreased again to the initial value (Fig. 6, uppermost panel). The corresponding instantaneous and averaged intensities of the $x$ - and $y$-polarized components versus time (and indirectly versus the injection current) are shown in Fig. 6. In Figure 7, the averaged polarization resolved intensities and frequencies are plotted versus the injection current (versus the thermal frequency-shift respectively) and are superimposed to the steady states (EC-mode structure) for the same parameters.

The averaged laser intensity is approximately equal to zero up to a current 
(a)

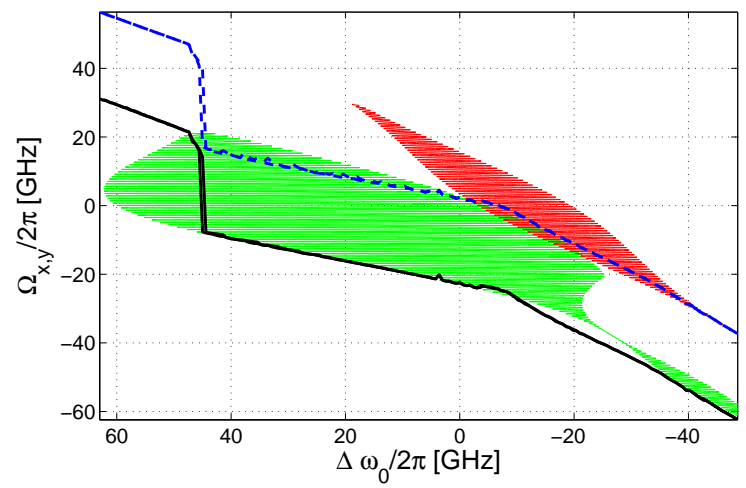

(b)

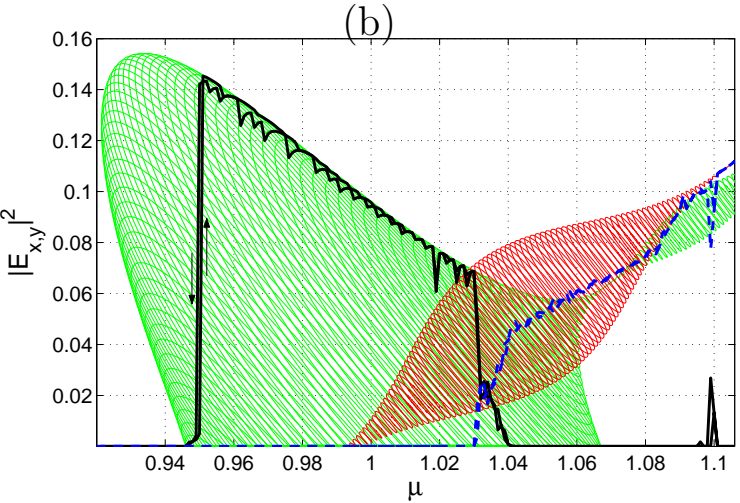

Fig. 7. Evolution of the averaged intensities $\left(<\left|E_{x}\right|>^{2}\right.$ and $\left.<\left|E_{y}\right|>^{2}\right)$ and of the averaged frequencies $\left(\left\langle\Omega_{x}\right\rangle\right.$ and $\left.\left\langle\Omega_{y}\right\rangle\right)$ for $E_{x^{-}}$and $E_{y^{-}}$polarized components versus injection current (b) or the solitary laser frequency $\Delta \omega_{0}$ (a). The thick solid and dashed curves denote the results obtained from numerical simulation for the $x$ - and $y$-component, respectively (in the online version the curves for the $x$ - and $y$-component are black and blue, respectively). The thin lines denote the analytically obtained steady-state solutions ( $x$ : light grey in print version, green in online version; $y$ : dark grey in print version, red in online version). The parameters are the same as in Figure 6.

value $\mu=0.95$ (we comment on the small pedestal at $\mu=0.95$ below). Then, the $x$-polarized component switches on rather abruptly to a high-amplitude state (Fig. 6, lowermost panel; Fig. 7b). After that, the intensity of the $x$ polarized component gradually decreases up to $\mu \approx 1.03$. At this point, the $y$ polarized component comes into play and there is strong competition between the two polarization components (see the oscillations in the instantaneous intensity in the two central panels of Fig. 6 ). At $\mu \approx 1.04$, the $x$-polarized component ceases to lase and the intensity of the $y$-polarized component continues to increase.

If the scan direction of the current is reversed, hysteresis phenomena are found in the time traces of the averaged intensities. First, the abrupt switch- 
off/switch-on of the high-amplitude $x$-polarized component shows hysteresis (below the solitary laser threshold). Second, in the region of a gradual change of the intensity of the $x$-polarized component $(0.95<\mu<1.03)$ hysteresis phenomena occur in conjunction with switching events between $x$-polarized EC-modes. We will interpret these findings in the following by comparing the numerically calculated LI-curve with the EC-mode set (Fig. 7).

Figure 7 shows that lasing $x$-polarized EC-modes appear at $\mu=0.92$, i.e., considerably below the threshold current value of $\mu=0.95$. At that point, a saddle-node bifurcation occurs in the phase space far above the zero intensity level resulting in the appearance of an EC-mode and an anti-mode. If the injection current is increased, more and more EC-modes arise and the region in phase space with EC-modes expands (the envelope resembling again part of an ellipse). It was checked that the EC-modes with maximal intensities in the upper part of the curve are stable. The nonlasing off-state is also stable up to the point where the lower part of the ellipse reaches the line of zero intensity and the anti-mode with the minimal intensity collides with the offstate. In between (i.e., $0.92 \lesssim \mu \lesssim 0.95$ ) there is bistability between the stable nonlasing state and stable lasing states of finite amplitude. The latter might be excited by an external hard perturbation (e.g., by additional optical injection) or possibly by a suitable change of control parameters. In the case of a gradual decrease of the injection current, the simulations show that the highamplitude states cannot be traced far below the switch-on point. The width of the hysteresis loop does not exceed the EC-mode spacing in the frequency domain, i.e., $\tau / k_{\mu}$ for the current, though it certainly exists.

Figure 7a shows that the switch-on of the laser is accompanied by a significant frequency shift of the laser (below the switch-on the frequency is the peak of the amplified spontaneous emission at the cavity resonance). At this point, the laser goes from freely running operation into the grating-controlled regime. The abrupt switch-on is reinforced due to positive feedback because of the phase-amplitude coupling (described by the $\alpha$-factor in (2)). Due to a small increase in intensity the carriers are weakly depleted. Hence, the refractive index increases and the cavity resonance red-shifts.As a consequence, the detuning decreases and the filter damps less strongly (if the detuning was initially positive), which causes an increase in intensity and closes the feedback loop.

Within the grating controlled regime for a single polarization component (e.g., for the $x$-component in the current interval between $0.95<\mu<1.03$, see Figs. 5 and $6 \mathrm{~b}$ ), multiple switching events between external cavity modes occur, if the current is scanned. A similar behavior is known for edge-emitting lasers [11]. For increasing current, the EC-mode become unstable due to a Hopf-bifurcation, for decreasing current due to a saddle-node. The transitions reflect the tendency of the laser to maximize output intensity, i.e., to minimize 
losses.

For $\Delta \omega_{0} /(2 \pi)<-20 \mathrm{GHz}$, the laser operates essentially as the solitary one. Here, the slope $d \Omega_{x} / d \Delta \omega_{0}$ is one, as expected. However, in the grating controlled regime, it is significantly smaller, about 0.3 indicating the relevance of frequency entrainment due to the filter. A similar tendency was found in the experiment, the frequency shift in the grating controlled regime (see Fig. 4) is about 0.4 of the shift for the solitary laser.

We commented already on the fact that the operating conditions of the two polarization modes depend strongly on the respective detunings of the polarization modes from the grating frequency as well as on the intrinsic and external polarization anisotropies. For the case under consideration, the $x$-polarized modes are favored at threshold. The threshold for the $y$-polarized mode is shifted by $\Delta \mu=0.05$ with respect to the one for the $x$-polarized mode. However, in the simulations excitation of the $y$-polarized mode does only occur at higher values of the pump, when the maximal intensity of the y-polarized ECmodes exceeds the one of the $x$-polarized modes (see Fig. $7 \mathrm{~b}$ ). This confirms again the tendency that the laser prefers the modes with the lowest losses. Slightly above this point, the orthogonally polarized modes oscillate in antiphase. Beginning from $\mu \approx 1.04$, only the $y$-polarized component is excited displaying complex oscillations up to $\mu \approx 1.09$ (Fig. 6, third panel). These occur presumingly since the feedback for the $y$-polarized component is strong in this current interval, resulting in coherence collapse and/or LFF. For higher currents, the system switches to stable $y$-polarized emission because the solitary laser frequency has left the feedback bandwidth. For a current value of $\mu \approx 1.1$, the effective feedback strength increases again for the $x$-polarized component in a small interval of current due to the fact that the solitary laser frequency is tuned to the secondary diffraction maximum of the grating. That leads to a weak excitation of the $x$-polarized component in the polarization resolved LI-curve. For higher currents, the thermal detuning from the grating frequency and the laser frequency is so high that the laser is essentially freely running. Here, the $y$-polarization is favored by the intrinsic anisotropies.

If the detuning between the frequency of the solitary laser at threshold and the grating frequency, $\Delta \omega_{0}^{t h}-\omega_{m}$, is decreased, the condition for optimal feedback for the $x$-polarization occurs for lower currents, i.e., the threshold decreases $(\mu=0.85$, Fig. 8a). Due to the lower injection, the amplitude of the emission reduces also. Apart from that, the main features observed are similar to the ones discussed before. A small pedestal is followed by an abrupt switchon of the $x$-component, which decreases again because the detuning becomes more unfavorable. The $x$-polarization decays already to zero (at $\mu=0.945$ ) before the threshold of the $y$-component is reached, i.e., the system has 'two thresholds'. At the second threshold, the $y$-component starts continuously (supercritical) from the off-state with a rather smooth intensity increase through 
(a)

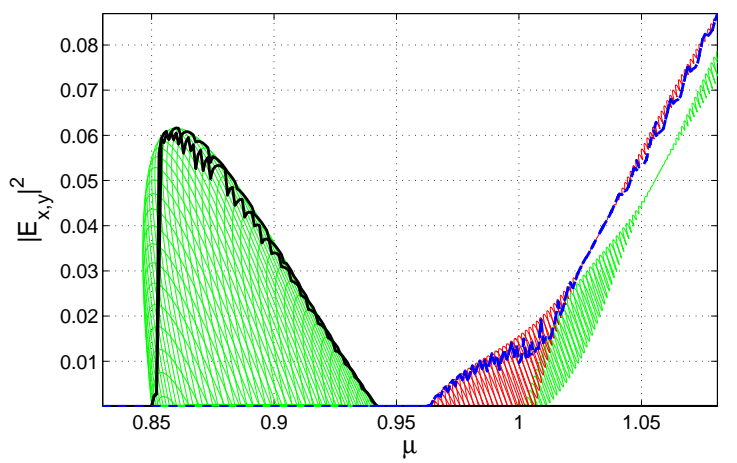

(b)

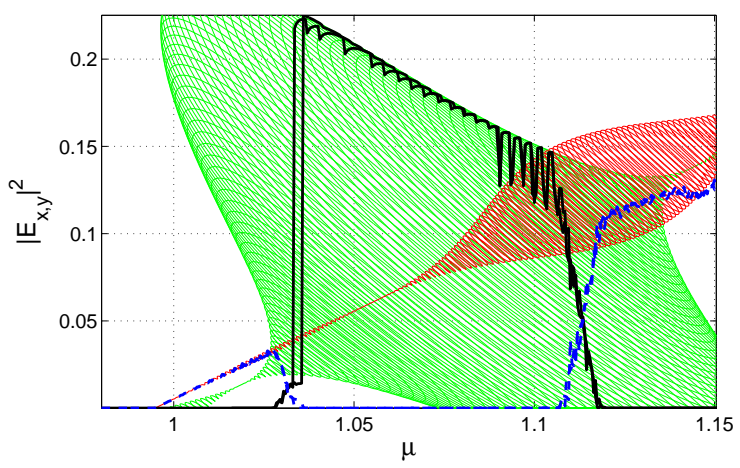

Fig. 8. The same as Fig. 7b, but $\Delta \omega_{0}^{\text {th }}=-60 \pi G H z$ (a), $120 \pi G H z$ (b).

a stable emission and, sometimes, almost regular oscillations. This can be interpreted as the normal switch-on of a laser slightly perturbed by feedback effects.

For an increase of the detuning, the threshold and the EC-mode structure is shifted in the direction of higher currents. Hence, in tendency, more ECmodes satisfy the lasing condition and the intensity of emission is increased. If the detuning is such (roughly for $\Delta \omega_{0}^{t h}-\omega_{m} \gtrsim 90 \pi \mathrm{GHz}$ ) that feedback is not significant before the threshold of the solitary laser is reached, the $y$-component arises at threshold because it has the lower intrinsic losses. An example is show in Fig. 8b for $\Delta \omega_{0}^{t h}-\omega_{m}=120 \pi G H z$. In that case, we observe a supercritical threshold behavior and a gradual increase of the intensity for the $y$-polarized component with a stable emission or rather regular oscillations $(0.995<\mu<1.027$, Fig. 8b). For higher pump currents $(1.027<\mu<1.035)$, the system demonstrates strong competition pulsations in the instantaneous intensities for both polarization components which have a LFF-like character. The time-average is again rather small leading to a small pedestal before the point of rapid switch-on of stable $x$-polarized emission.

After the switch-on, a gradual decrease in the intensity of the x-polarized component is observed, as for the detuning values considered before. Above 
$\mu=1.105$, competition between the two polarization components sets in; and for current values above $\mu=1.12$ only the $y$-polarized component is active displaying coherence collapse.

The scenario shown in Fig. 8a for small detuning matches the experimental observations for the corresponding situation (Fig. 3) rather closely. In both cases there is an abrupt switch-on to a high-amplitude state, which shows a small hysteresis. Furthermore, the existence of bistability is indicated in the experiment by the erratic, probably noise-induced, jumps of high amplitude. After the abrupt switch-on, the intensity of the $x$-polarized mode (the weaker mode in the solitary laser) decreases gradually. Afterwards, the $x$-polarized mode is essentially off, as in the solitary laser and then lasing takes place only in the $y$-component (not shown in the experiment). Ripples in the LIcurves are also present in the experimentally obtained LI-curves (Figs. 2, 3). In tendency, the up-scan has a higher amplitude than the down-scan matching the observations in the simulations. Figure 4 indicates that the ripples are connected to jumps in frequency. This supports their interpretation as jumps between different EC-modes.

Our experimental detection system allowed only the measurement of the average intensity and hence we can not strongly comment on the possibility of self-oscillations obtained in numerical simulations before the switch-on to a stable high-intensity mode. However the experimentally recorded LI-curve (Fig. 3) shows a small pedestal before the abrupt switch-on being very similar to the remnant of the high-amplitude oscillations in the time-averaged numerical curves (Fig. 6, lowest panel, Fig. 7b). Hence, it is possible that self-oscillations were present also in the experiment.

A closer inspection of the system behavior in this range of currents (see Fig. 6, second panel) reveals that when the off-state is destabilized, the system displays pulsations grouped in packages. Each group consists of a train of short spikes equally spaced by the external cavity delay time $\tau$. The amplitude of the pulses can strongly exceed the steady state value of the EC-modes existing for the same parameters (by 10 times in our simulations), but the averaged intensity is more than 10 times lower than the steady state values due to the short duration of the spikes. The distance between the pulse packages decreases from several hundreds of ns to several tens of ns with increasing current. These oscillations appear presumingly due to the mechanism described in [26], i.e., a synchronization of the EC-modes. In conjunction with a low-frequency pulse envelope these form a synchronized torus-like state $[27,28]$ with a symmetrical shape. This torus-like state is gradually deformed to asymmetrical low-frequency oscillations (LFF) with pronounced intensity dropout for an increase of the injection current as in VCSELs with isotropic optical feedback [28]. The interval of the injection current where the system is oscillating depends on the level of spontaneous emission noise, but was checked to occur 
also in neighboring parameter regions (e.g., we checked for a lower number of the $\alpha$-factor, $\alpha=3$ ). The question, whether a direct switching from the off-state to a stable EC-mode can take place, needs to be addressed in further investigations.

\subsection{Feedback conditions favoring the stronger polarization modes}

Here, we are going to consider briefly the inverse relation of the feedback strengths, i.e., the case that the feedback is favoring the $y$-polarized component having also the lower losses in the solitary laser.

For zero and negative detunings, $\Delta \omega_{0}^{t h}-\omega_{m} \lesssim 0$, we observe only the $y$ polarized component in the LI-curves (Fig. 9a). After an abrupt switch-on, the intensity of the $y$-polarized component decreases again and reaches a local minimum around $\mu \approx 1.025$. Afterwards, the intensity increases again representing mainly the emission of the solitary laser, weakly perturbed by the feedback arising from the secondary diffraction maximum of the grating for $1.03 \lesssim \mu \lesssim 1.12$. This LI-curve closely resembles the experimentally one for the corresponding case (Fig. 2).

For values of the detuning between $30 \pi \mathrm{GHz}$ and $60 \pi \mathrm{GHz}$, low amplitude $x$-polarized emission appears supercritically before the abrupt switch-on of the $y$-polarized component, because it is favored by birefringence in that region. For even higher values of the detuning (e.g., $90 \pi \mathrm{GHz}$, Fig. 9b), the $y$-component appears supercritically in the vicinity of the threshold of the solitary laser (there is a small threshold reduction due to feedback arising from the secondary diffraction maximum of the grating). For somehow higher values of the current, there is competition between the two polarization components (the $y$-component being favored by the intrinsic amplitude anisotropies and the $x$-component experiencing feedback due to the matching birefringence) followed by an abrupt switch-on of a high-amplitude $y$-polarized steady state (Fig. 9b). At this point, there is bistability between a stable $y$-polarized ECmode and polarization pulsations leading to hysteresis. The bistability between solitary laser states and EC-modes was reported also for the scalar case in edge-emitting lasers, e.g. [9].

\section{Summary and conclusion}

In this paper, we investigated the behaviors of VCSELs with frequency-selective feedback. We obtained the possibility of a very abrupt turn-on to a highamplitude state with hysteresis. After this switch-on, the emitted intensity 
(a)

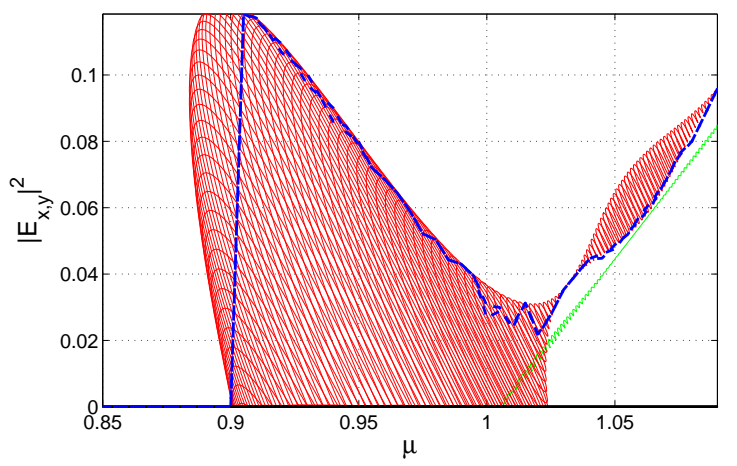

(b)

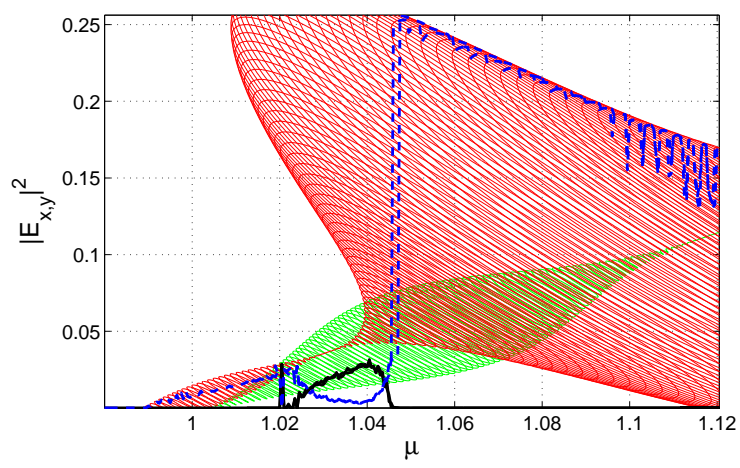

Fig. 9. The same as Fig. 7b,but $\sigma_{x}=10 n s^{-1}, \sigma_{y}=60 n s^{-1}, \Delta \omega_{0}^{t h}=-60 \pi G H z$ (a), $90 \pi G H z$ (b).

typically decreases till the threshold of the solitary laser is reached. For high enough currents, the laser is always operating more or less uninfluenced by the feedback. This is related to the thermal frequency shift with current responsible for the fact that feedback can be effective only in a small range of currents.

The experimental observations are in good agreement with expectations from theory based on a vectorial model for the laser dynamics. The investigations yield bistability between the stable non-lasing off-state and stable highamplitude EC-modes. This bistability leads to the abrupt turn-on when there is the positive feedback between the detuning conditions of the laser frequency and the grating frequency and phase-amplitude coupling. In the simulations (and probably in the experiment) the switch-on is preceded by a small region with oscillations. However, it should be possible to switch directly from the off-state to the high-amplitude lasing states by external, hard perturbations. Hence, this scheme has potential for all-optical photonic switching. In particular, a version of this experiment using a broad-area laser is attractive for the investigation of the possibility of cavity solitons [29].

Threshold conditions and the competition between the two polarization modes 
are influenced by many parameters, especially the intrinsic and external amplitude anisotropies and the difference in detuning conditions brought about by birefringence. We described and analyzed the resulting transition scenarios in detail including the possibility of switching events between external-cavity modes and the resulting hysteresis in amplitude and frequency. In tendency, it was found that the transition between different EC-modes (with the same or a different polarization) could be understood by a preference for the mode with the lowest losses and hence highest intensity. Within the current intervals where one of the polarization modes is dominating, some regularities of the VCSEL behavior are similar to those for edge-emitting semiconductor lasers (see for example [6-12]). However, the abrupt switch-on at threshold obtained in the present work were not demonstrated for those lasers, possibly, since it is difficult to realize such a strong feedback in edge-emitting lasers.

\section{Acknowledegments}

This work was supported by the Deutsche Forschungsgemeinschaft by travel grants and money for equipment.

\section{A Appendix}

\section{A.1 Transfer function of the grating}

The transfer function of the grating can be obtained from the following considerations: If $x=T^{\prime}\left(\Omega-\omega_{m}\right)$ is a normalized detuning from the frequency experiencing optimal feedback in the first diffraction order, $u^{(0)}(x)$ the transfer function of a one groove (periodicity interval) of the grating and $M$ the number of the illuminated grooves, then the general transfer function of the grating is

$$
u(x)=u^{(0)}(x) \frac{1}{M} \sum_{j=0}^{M-1} \exp (-i j x)=u^{(0)}(x) \frac{1}{M} \frac{1-\exp (-i M x)}{1-\exp (-i x)} .
$$

For large $M$ and for small detuning considered in the paper (i.e., $x \ll$ frequency distance between adjacent diffraction orders) yields

$$
u(x) \simeq \text { const } \times \exp (-\mathrm{ixM} / 2) \frac{\sin \mathrm{xM} / 2}{\mathrm{xM} / 2} .
$$


The grating bandwidth $1 / T=2 /\left(T^{\prime} M\right)$ is related to the half width at half maximum, HWHM, of the double-reflection transfer function $h(\Omega)^{2}$ (6) by $T=x_{0} /$ HWHM, where $x_{0}$ is the root of the equation $\sqrt[4]{2} \sin x_{0}=x_{0}$ and $x_{0} \simeq 1.0019$.

\section{A.2 Steady states}

Steady states solutions (monochromatic solutions) of the system (1)-(5) in the absence of noise are found in the form $E_{ \pm}=Q_{ \pm} e^{ \pm i \psi+i \Omega t}, N=N_{s}$ and $n=n_{s}$. We present here only linearly polarized states since they are relevant to our investigations.

There are two types of linearly polarized solutions (with $\mathrm{x}$ - and $\mathrm{y}$ - polarization orientations), which are denoted by $\hat{x}$ - and $\hat{y}$ - states. Their frequencies are determined from the following transcendent equations:

$$
\left(-\alpha \gamma_{a}+\gamma_{p}\right)(-1)^{l}+\Omega-\Delta \omega_{0}+\sigma_{x, y}[\alpha \operatorname{Re} f(\Omega)-\operatorname{Im} f(\Omega)]=0,
$$

where $l=0(1)$ and $\sigma_{x}\left(\sigma_{y}\right)$ corresponds to $\hat{x}_{-}\left(\hat{y}^{-}\right)$polarized states and

$$
f(\Omega)=h(\Omega)^{2} \exp \left(-i\left(\Omega+\omega_{0}\right) \tau\right)=H(\Omega)^{2} \exp (-i \phi),
$$

with the normalized absolute value (or the shape) of the filter reflectivity $H(\Omega)^{2}=$

$\left(\sin T\left(\Omega-\omega_{m}\right) /\left(T\left(\Omega-\omega_{m}\right)\right)\right)^{2}$ and the phase factor $\phi=-2 T\left(\Omega-\omega_{m}\right)-\tau(\Omega+$ $\left.\omega_{0}\right)$. The steady inversion is $N_{s}=1+\frac{1}{k}\left((-1)^{l} \gamma_{a}-\sigma_{x, y} \operatorname{Re} f(\Omega)\right), n_{s}=0$;

the field amplitudes are given by $Q_{ \pm}=\sqrt{\frac{1}{2}\left(\frac{\mu}{N_{s}}-1\right)}$ and $\psi=l \pi / 2$.

The threshold value for the injection current for each linearly polarized state is

$\mu_{t h}=N_{s}=1+\frac{1}{k}\left((-1)^{l} \gamma_{a}-\sigma_{x, y} \operatorname{Re} f(\Omega)\right)$.

In the plane spanned by frequency-inversion $(\Omega, N)$ (or by frequency-intensity $\left.\left(\Omega,|E|^{2}\right)\right)$, the $\hat{x}$ - and $\hat{y}$ - linearly polarized states lie on two ellipse-like curves (with frequency separations between the ellipses approximately equal to $2\left(\gamma_{p}-\alpha \gamma_{a}\right)$ ).

Near the solitary laser threshold, only those parts of these ellipses exist which satisfy the threshold condition: $\mu \geq \mu_{t h}=N_{s}(\Omega)$. The lower branch of each ellipse in the $(\Omega, N)$-diagram (or the upper branch in the $\left(\Omega,|E|^{2}\right)$ )-diagram) consists of external cavity modes (or nodes resulting from constructive interference). The upper branch in the $(\Omega, N)$-diagram (the lower branch in the $(\Omega$, $\left.|E|^{2}\right)$ )-diagram) consists of external cavity anti-modes (saddles which result from destructive interference). 
In the system, there is also a trivial non-lasing steady-state with zero intensity and $N_{s}=\mu$.

\section{A.3 Linear stability analysis}

\section{A.3.1 Linearized equations.}

$a_{ \pm}$and $\Delta_{ \pm}$are taken to be small deviations from steady solutions $\left(\epsilon_{ \pm}, N_{ \pm}^{s}\right)$ : $E_{ \pm}=\left(\epsilon_{ \pm}+a_{ \pm}\right) e^{i \Omega t}$ and $N_{ \pm}=N_{ \pm}^{s}+\Delta_{ \pm}$(below index " $s "$ will be omitted). Then the linearization of the system (1) near these solutions gives:

$$
\begin{aligned}
\bar{a}^{\prime} & =(\hat{G}-i \Omega \hat{I}) \bar{a}+\hat{\xi} \bar{\Delta}+\hat{\sigma} \mathrm{E} \cdot \bar{a}, \\
\bar{\Delta}^{\prime} & =\hat{\zeta} \bar{a}+\text { c.c. }+\hat{u} \bar{\Delta}
\end{aligned}
$$

where $\bar{a}=\left(a_{+}, a_{-}\right)$and $\bar{\Delta}=\left(\Delta_{+}, \Delta_{-}\right)$are vector columns and

$\hat{\xi}=\left(\begin{array}{ll}s \epsilon_{+} & 0 \\ 0 & s \epsilon_{-}\end{array}\right), \hat{\zeta}=-2\left(\begin{array}{cc}N_{+} \epsilon_{+}^{*} & 0 \\ 0 & N_{-} \epsilon_{-}^{*}\end{array}\right), \hat{u}=-\frac{1}{2}\left(\begin{array}{cc}1+\gamma_{s}+4 Q_{+}^{2} & 1-\gamma_{s} \\ 1-\gamma_{s} & 1+\gamma_{s}+4 Q_{-}^{2}\end{array}\right)$,

and $\mathrm{E}$ is defined as: $\mathrm{\epsilon} \cdot a(t) \equiv \frac{1}{2 \pi} \int_{-\infty}^{+\infty} d \omega f(\omega) \int_{-\infty}^{+\infty} d t^{\prime} \exp \left(i(\omega-\Omega)\left(t-t^{\prime}\right)\right) a\left(t^{\prime}\right)$ Then the stability of the steady solutions of the system (1) is governed by the roots of the following equation: $\operatorname{det}(\mathbf{A}+\mathbf{B}(z)-\mathbf{E} z)=0$, where
$\mathbf{A}=\left(\begin{array}{llr}\hat{G}-i \Omega \hat{I} & \hat{0} & \hat{\xi} \\ \hat{0} & \hat{G}^{*}+i \Omega \hat{I} & \hat{\xi}^{*} \\ \hat{\zeta} & \hat{\zeta}^{*} & \hat{u}\end{array}\right)$,
, $\mathbf{B}(z)=\left(\begin{array}{l}\hat{\sigma} f \\ \hat{0} \\ \hat{0}\end{array}\right.$
$\left.\begin{array}{lr}\hat{0} & \hat{0} \\ \hat{\sigma} f^{*}(\Omega-i z) \hat{I} & \hat{0} \\ \hat{0} & \hat{0}\end{array}\right)$,

and $\hat{0}$ is $(2 \times 2)$ zero matrix, $\hat{I}$ and $\mathbf{E}$ are $(2 \times 2)$ and $(6 \times 6)$ identity matrices.

\section{A.3.2 Linearly polarized states.}

For linearly polarized states ( $\hat{x}$ - and $\hat{y}$-states), the linearized system (A.4) is separated in two independent subsystems for the cartesian variables $a_{x, y}$ and $(\Delta, \delta)$, with $a_{ \pm}=\left(a_{x} \pm a_{y}\right) / 2$ and $\Delta_{ \pm}=\Delta \pm \delta$. One of these subsystems describes the stability of the steady state to perturbations with the same polarization, the other one - its stability to perturbations with the orthogonal polarization. E.g., for $\hat{x}$ - states the subsystems are:

$$
\begin{aligned}
\dot{a}_{x, y} & =\left[s(N-1)+i\left(\Delta \omega_{0}-\Omega\right) \pm \gamma\right] a_{x, y}+2 s Q \Delta_{x, y}+\sigma_{x, y} \mathrm{E} \cdot a_{x, y} \\
\dot{\Delta}_{x, y} & =-N Q a_{x, y}+\text { c.c. }-\left(\gamma_{x, y}+2 Q^{2}\right) \Delta_{x, y}
\end{aligned}
$$

where $\Delta_{x, y} \equiv(\Delta, \delta)$ and $\gamma_{x, y} \equiv\left(1, \gamma_{s}\right)$. 


\section{A.3.3 Off-state.}

For the nonlasing off-state, the above linearization gives (we consider field deviations in the form $E_{ \pm}=a_{ \pm}$for the case):

$$
\begin{aligned}
\dot{a}_{x, y} & =\left[s(\mu-1)+i \Delta \omega_{0} \pm \gamma\right] a_{x, y}+\sigma_{x, y} \mathrm{E} \cdot a_{x, y}, \\
\dot{\Delta}_{x, y} & =-\gamma_{x, y} \Delta_{x, y} .
\end{aligned}
$$

Then, characteristic exponents (roots) $z$ for the field deviations can be obtained from the following equations:

$$
s(\mu-1)+i \Delta \omega_{0} \pm \gamma-z+\sigma_{x, y} f(-i z)=0 .
$$




\section{References}

[1] F. Favre and D. Le Guen, IEEE J. of Quantum Electron., vol. QE-21, pp. 1937-1946, 1985.

[2] R. Wyatt and W.J. Delvin, Electron Lett., vol. 19, pp.110-112, 1983.

[3] E. Brinkmeyer, W. Brennecke, M. Zurn, and R Ulrich, Electron. Lett., vol. 22, pp. 134-135, 1986.

[4] M. Kozuma, M. Kourogi, and M. Ohtsu, Appl.Phys.Lett., vol. 61, pp.1895-1897, 1992.

[5] M.W. Fleming, and A. Mooradian, IEEE J. of Quantum Electronics, vol. QE17, pp.44-59, 1981.

[6] P. Zorabedian, W.R. Trutna, and L.S. Cutler, IEEE J. of Quantum Electronics, vol. QE-23, pp.1855-1860, 1987.

[7] P. Zorabedian, IEEE J. of Quantum Electronics, vol. QE-30, pp.1542-1552, 1994.

[8] Chi Yang, X. Wang, and J.G. McInerney, IEEE J. of Quantum Electronics, vol. QE-232, pp.813-821, 1996.

[9] M. Giudici, L. Giuggioli, C. Green, and J.R. Tredicce, Chaos, Solitons and Fractals, vol. 9, pp.1-8, 1998.

[10] M. Yousefi and D. Lenstra, IEEE J. of Quantum Electronics, vol. QE-35, pp.970976, 1999.

[11] A.P.A. Fischer, O.K. Andersen, M. Yousefi, S. Stolte and D. Lenstra, IEEE J. of Quantum Electronics, vol. QE-36, pp.375-384, 2000.

[12] A. Naumenko, P. Besnard, N. Loiko, G. Ughetto and J.C. Bertreux, IEEE J. of Quantum Electron, vol. 39, pp.1216-1228, 2003.

[13] C.J. Chang-Hasnain, J.P. Harbison, G. Hasnain, A. von Lehmen, L.T. Florez, and N.G. Stoffel, Electron Letts. vol. 27, pp.163-165, 1991.

[14] B. Ryvkin, K. Panajotov, A. Georgievskii, J. Danckaert, M. Peeters, G. Verchaffelt, H. Thienpont, and I. Veretennicoff, J. Opt. Soc. Am. B, vol. B 16, pp.2106-2113, 1999.

[15] M. B. Willemsen, M.P. van Exter, and J.P. Woerdman, Phys.Rev. Lett., vol. 84, pp.4337-4340, 2000.

[16] T. Ackemann and M. Sondermann, Appl. Phys. Letts., vol. 78, pp.3574-3576, 2001.

[17] S. Jiang, Z. Pan, M. Dagenais, R.A. Morgan, and K. Kojima, Appl. Phys. Lett., vol. 63, pp.3545-3547, 1993. 
[18] F. Robert, P. Besnard, M.-L. Chares, and G.M. Stephan, Opt. Quantum Electron, vol. 27, pp. 805-811, 1995; P. Besnard, F. Robert, M.-L. Chares, and G.M. Stephan, Phys. Rev. A, vol. 56. pp. 3193-3205, 1997.

[19] N.A. Loiko, A.V. Naumenko, and N.B. Abraham, Quantum Semiclass. Opt., vol. 10, pp.125-140, 1998; J. Opt. B: Quantum Semiclass. Opt., vol. B3, pp.S100S111, 2001.

[20] C.I. Wilkinson, J. Woodhead, J.E.F. Frost, J.S. Roberts, R. Wilson, and M.F. Lewis, IEEE Photon. Technol. Lett. vol. 11, pp.155-157, 1999.

[21] Y. Hong, P.S. Spencer, and K.A. Shore, Optics. Lett., vol. 29, pp.2151-2153, 2004 .

[22] P. Debernardi, J.M. Ostermann, M. Feneberg, C. Jalics, R. Michalzik, IEEE Journal of Selected Topics in Quantum Electronics, vol. 11, pp. 107-115, 2005.

[23] S. Barland, J.R. Tredicce, M. Brambilla, L.A. Lugiato, S. Balle, M. Giudici, T. Maggipinto, L. Spinelli, G. Tissoni, T. Knodel, M. Miller, R. Jager, Nature, vol. 419, pp. 699-702, 2002.

[24] F. Marino, S. Barland, and S. Balle, IEEE Photon. Technol. Lett. vol. 15, pp.789-791, 2003.

[25] M. S.Miguel, Q. Feng and J. V.Moloney, Phys. Rev. A , vol. A52, pp.1728-1739, 1995.

[26] P. Besnard, B. Mezaine, and G.M. Stephan, IEEE J. Quantum Electron. vol. 29, pp.1271-1284, 1993.

[27] R.L. Davidchack, Y.-C. Lai, A. Gavrielides, and V. Kovanis, Phys. Rev. E, vol. E63, 056206, 2001.

[28] A.V. Naumenko, N.A. Loiko, M. Sondermann, and T. Ackemann, Phys. Rev. A, vol. A68, 033805, 2003.

[29] M. Sondermann, F. Marino, K. F. Jentsch, T. Ackemann, and R. Jäger. In Nonlinear Guided Waves and Their Applications, Toronto, March 28-31, 2004. Paper MB5. 


\section{List of Figure Captions}

Fig. 1. Experimental setup: CL collimating lens, $\lambda / 2$ half-wave retardation plate, G grating, M HR-mirror, ISO optical isolator, BS beam splitter, D lowbandwidth detector, FPI scanning Fabry-Perot interferometer.

Fig. 2. Output power for increasing injection current. The anisotropies of the external cavity are optimized for feedback favoring the polarization component already dominant in the free-running laser. The grey line denotes the free running laser, the black one the laser with feedback. The inset shows an enlargement of the region around threshold. The detuning is optimized to achieve a large response in the vicinity of the threshold.

Fig. 3. Output power for increasing injection current. The anisotropies of the external cavity are optimized for feedback favoring the polarization component disfavored in the free-running laser. The grey line denotes the free running laser, the black solid line the laser with feedback for increasing current, the black dotted line the laser with feedback for decreasing current. The detuning is optimized to achieve a large response in the vicinity of the threshold.

Fig. 4. Emission frequency versus current in the grating controlled regime of Fig. 3.

Fig. 5. Steady states (external cavity modes) in the domain frequency-amplitude (lower panels) without frequency-filtering effects (a) and with frequency-filtering from the external diffraction grating (b) and corresponding transfer functions (upper panels). Parameters: $\kappa=300 n s^{-1}, \alpha=5, \gamma_{a}=0.5 \pi n s^{-1}$, $\gamma_{p}=25 \pi n s^{-1}, \gamma_{s}=40 n s^{-1}, \mu=1, \tau=1.4 n s, \sigma_{x}=60 n s^{-1}, \sigma_{y}=10 n s^{-1}$, $H W H M=24 \pi n s^{-1}, \Delta \omega_{0}=0, \omega_{m}=0$.

Fig. 6. Time evolution of instantaneous intensities of $E_{x^{-}}$and $E_{y^{-}}$polarized components $\left(\left|E_{x}\right|^{2}\right.$ and $\left|E_{y}\right|^{2}$, central panels) and of averaged intensities $\left(<\left|E_{x}\right|>^{2}\right.$ (solid curve) and $<\left|E_{y}\right|>^{2}$ (dashed curve), lowermost panel), when the injection current $\mu$ is scanned (uppermost panel). Parameters: $\beta=10^{-5}, \Delta \omega_{0}^{t h}=30 \pi G H z, k_{\mu}=600 G H z$. Other parameters are the same as in Figure 5.

Fig. 7. Evolution of the averaged intensities $\left(<\left|E_{x}\right|>^{2}\right.$ and $\left.<\left|E_{y}\right|>^{2}\right)$ and of the averaged frequencies $\left(<\Omega_{x}>\right.$ and $<\Omega_{y}>$ ) for $E_{x^{-}}$and $E_{y^{-}}$polarized components versus injection current (b) or the solitary laser frequency $\Delta \omega_{0}$ (a). The thick solid and dashed curves denote the results obtained from numerical simulation for the $x$ - and $y$-component, respectively (in the online version the curves for the $x$ - and $y$-component are black and blue, respectively). The thin lines denote the analytically obtained steady-state solutions $(x$ : light grey in print version, green in online version; $y$ : dark grey in print version, red in 
online version). The parameters are the same as in Figure 6.

Fig. 8. The same as Fig. 7b, but $\Delta \omega_{0}^{t h}=-60 \pi G H z$ (a), $120 \pi G H z$ (b).

Fig. 9. The same as Fig. 7b,but $\sigma_{x}=10 n s^{-1}, \sigma_{y}=60 n s^{-1}, \Delta \omega_{0}^{t h}=-60 \pi G H z$ (a), $90 \pi G H z$ (b). 